\title{
APLICAÇÃO DA METODOLOGIA DE SUPERFÍCIE DE RESPOSTA PARA O ESTUDO DO EFEITO DA TEMPERATURA SOBRE O COMPORTAMENTO REOLÓGICO DE MISTURAS TERNÁRIAS DE POLPA DE MANGA E SUCOS DE LARANJA E CENOURA ${ }^{1}$
}

\author{
Ivanise Guilherme BRANCO ${ }^{2, *}$, Carlos Alberto GASPARETTO ${ }^{3}$
}

\begin{abstract}
RESUMO
Neste trabalho estudou-se o comportamento reológico da mistura ternária de polpa de manga e sucos de laranja e cenoura, nas temperaturas de 10 e $60^{\circ} \mathrm{C}$. As proporções dos componentes foram determinadas através da metodologia de superfície de resposta para mistura. As medidas experimentais foram realizadas no reômetro Haake Rotovisco RV-20 e as curvas de escoamento foram ajustadas ao modelo de Ostwald- De Waele. Os parâmetros reológicos foram utilizados como resposta no delineamento e verificou-se que os parâmetros indice de consistência e indice de comportamento do fluido, nas duas temperaturas, foram melhores ajustados com os modelos linear e quadrático, respectivamente.

Palavras-chave: reologia; polpa de manga; suco de laranja; suco de cenoura; misturas ternárias.
\end{abstract}

\section{SUMMARY}

RESPONSE SURFACE MATHODOLOGY APPLIED TO THE STUDY OF TEMPERATURE EFFECT ON THE RHEOLOGICAL BEHAVIOR OF TERNARIES MIXTURES WITH MANGO PULP AND ORANGE AND CARROT JUICES.In this work was studied the rheological behaviour of ternaries mixtures of pulp mango, orange and carrot juices, in temperatures of 10 and $60^{\circ} \mathrm{C}$. The juice proportion were defined by response surface methodology to mixture. Rheological data were obtained in a Haake Rotovisco model RV 20 and rheograms were fitted with OstwladDe Waele. The rheological parameters were used as response for the experimental designs. It was observed that the parameters consistency index and flow behaviour index, in the two temperatures, were better described with the linear and quadratic model, respectively.

Keywords: rheology; mango pulp; orange juice; carrot juice; ternaries mixtures.

\section{1 - INTRODUÇÃO}

Atualmente há um mercado crescente para sucos compostos com mais de uma fruta e essa tendência é mais observada em produtos que utilizam em suas formulações frutas tropicais. A mistura de sucos de laranja e cenoura com polpa de manga foi definida por ser esta última portadora de polpa atrativa e sabor exótico, além de vários nutrientes como beta caroteno, ácido ascórbico, sais minerais e considerável teor de fibra. Já o suco de laranja foi escolhido por possuir sabor conhecido, ter ampla aceitabilidade e grande disponibilidade, enquanto que o suco de cenoura é altamente rico em carotenóides, tem cor compativel com os outros dois e não afeta desfavoravelmente a aceitabilidade do produto.

$\mathrm{Na}$ caracterização reológica desses sucos foi utilizado o modelo reológico de Ostwald- De Waele (equação 1) por ajustar-se bem aos dados experimentais, ser um modelo simples e de ampla aplicação tecnológica.

$\tau=\mathrm{K} * \gamma^{\mathrm{n}}$

onde $\tau$ é a tensão de cisalhamento, $\gamma$ é a taxa de deformação, K é o índice de consistência e $\mathrm{n}$ o índice de comportamento do fluido.

\footnotetext{
1. Recebido para publicação em 17/09/2002. Aceito para publicação em 28/07/2003 (000988).

${ }^{2}$ Universidade Estadual do Centro-Oeste (UNIOCENTRO), Depto. de Engenharia de Alimentos, Cx.P. 730, Guarapuava, PR.E-mail: wanise@unicentro.br ${ }^{3}$ Universidade Estadual de Campinas, Depto. de Engenharia de Alimentos, CEP 13080-970, Campinas, SP.E-mail: gasparetto@ceres.fea.unicamp.br * A quem a correspondência deve ser enviada.
}

Alguns autores estudaram o comportamento reológico de polpa de manga: MANOHAR, RAMAKRISHNA \& RANTEKE [9], SAEED [17], GUNJAL \& WAGHMARE [7], VIDAL [18, 19], QUEIROZ [13] e PELEGRINE [12]. Todos encontraram comportamento pseudoplástico para este tipo de suco. LOMBRAÑA \& DIAS [8] estudaram as mudanças reológicas em suco de cenoura centrifugado e verificaram comportamento pseudoplástico. Vários autores estudaram o comportamento reológico do suco de laranja concentrado: MIZHARI \& BERK [10]; MIZHARI \& FIRSTENBERG [11]; CRANDALL, CHEN \&CARTER [6]; VITALI [20]; RAO, COOLEY \& VITALI [15], RAO et al. [16] e BRANCO [4]. Todos estes autores encontraram comportamento pseudoplástico para o suco de laranja concentrado e newtoniano para o natural. A combinação da polpa de manga com os sucos de laranja e cenoura levará a um produto com comportamento ainda não descrito na literatura, necessitando assim de uma criteriosa caracterização.

Portanto o objetivo do trabalho foi estudar o comportamento reológico da mistura de sucos de laranja e cenoura com polpa de manga, nas temperaturas de 10 e $60^{\circ} \mathrm{C}$ utilizando-se a metodologia de superfície de resposta para mistura para descrever a influência de cada componente sobre os parâmetros reológicos.

\section{2 - MATERIAL E MÉTODOS}

\section{1 - Matérias-primas}

As mangas, da variedade "Haden", foram despolpadas e armazenadas a $-20^{\circ} \mathrm{C}$. As cenouras, da variedade "Nantes", foram trituradas e prensadas para obtenção do suco. O suco de laranja utilizado neste trabalho foi o concentrado congelado comercial sem adição de açú- 
car, proveniente de um mesmo lote de processamento. O suco foi diluído em água destilada a $12^{\circ}$ Brix para ser misturado com os outros sucos.

\subsection{1 - Caracterização da matéria-prima}

Foram determinados, na polpa de manga e nos sucos de laranja e cenoura, o teor de sólidos solúveis ( ${ }^{\circ}$ Brix) medido diretamente em um refratômetro de bancada (marca Warszawar, modelo RL3); $\mathrm{pH}$ leitura direta em potenciômetro (marca Mettler Toledo, modelo 320); acidez titulável, utilizando o método 22038 da A.OA.C. [1]; pectina, empregando o método de Carré \& Haynes [14]; açúcares redutores e totais usando o método titrimétrico baseado na redução de cobre pelos grupos redutores dos açúcares, segundo Lane-Eynon [14]; fibras determinadas pelo método enzimático descrito por ASP et al. [2] e teor de polpa, por centrifugação.

\section{2 - Medidas reológicas}

No estudo reológico utilizou-se o reômetro Haake Rotovisco RV-20 e o sistema de medida adotado foi o de placas paralelas PQ $45 \mathrm{com}$ distanciamento de $0,5 \mathrm{~mm}$. Os dados reológicos foram obtidos em triplicata. As medidas foram realizadas nas temperaturas de 10 e $60^{\circ} \mathrm{C}$ por serem, respectivamente, a temperatura típica de prateleira do produto acabado e a temperatura representativa de pasteurização nas indústrias.

\section{3 - Formulação e delineamento experimental}

As proporções das misturas foram definidas empregando-se a metodologia de superfície de resposta para mistura [5]. Neste trabalho a polpa de manga estará presente em 60\%, no mínimo, para cada formulação, enquanto que para os outros a restrição será de 10\%, no mínimo. Na Tabela 1 encontram-se as 10 combinações usadas, tanto em termos de componentes originais como pseudo-componentes. Este último é definido como combinação do componente original.

TABELA 1. Delineamento simplex centróide aumentado de 10 tratamentos para as misturas com polpa de manga e sucos de laranja e cenoura.

\begin{tabular}{c|cccc|ccc}
\hline \multirow{2}{*}{ Formulação } & \multicolumn{3}{|c|}{ Pseudo-componentes } & \multicolumn{3}{c}{ Componentes Originais } \\
\cline { 2 - 7 } & $\mathrm{X}^{\prime} 1$ & $\mathrm{X} 2$ & $\mathrm{X} 3$ & $\mathrm{X} 1$ & $\mathrm{X} 2$ & $\mathrm{X} 3$ \\
\hline 1 & 1 & 0 & 0 & 0.8 & 0.1 & 0.1 \\
2 & 0 & 1 & 0 & 0.6 & 0.3 & 0.1 \\
3 & 0 & 0 & 1 & 0.6 & 0.1 & 0.3 \\
4 & $1 / 2$ & $1 / 2$ & 0 & 0.7 & 0.2 & 0.1 \\
5 & $1 / 2$ & 0 & $1 / 2$ & 0.7 & 0.1 & 0.2 \\
6 & 0 & $1 / 2$ & $1 / 2$ & 0.6 & 0.2 & 0.2 \\
7 & $1 / 3$ & $1 / 3$ & $1 / 3$ & 0.6666 & 0.16666 & 0.16666 \\
8 & $2 / 3$ & $1 / 6$ & $1 / 6$ & 0.73333 & 0.13333 & 0.13333 \\
9 & $1 / 6$ & $2 / 3$ & $1 / 6$ & 0.63333 & 0.23333 & 0.13333 \\
10 & $1 / 6$ & $1 / 6$ & $2 / 3$ & 0.63333 & 0.13333 & 0.23333 \\
\hline X1 = polpa de manga; X2 = suco de laranja; X3 $=$ suco de cenoura & \\
Restrição: X1 $\geq 60, \mathrm{X} 2 \geq 10$, X3 $\geq 10$ &
\end{tabular}

Para encontrar a proporção original dos três componentes, transformou-se os dados usando as seguintes fórmulas:

$$
X_{1}^{\prime}=\frac{X_{1}-0,6}{0,2} \quad X_{2}^{\prime}=\frac{X_{2}-0,1}{0,2} \quad X_{3}^{\prime}=\frac{X_{3}-0,1}{0,2}
$$

$\mathrm{Na}$ representação do ajuste dos valores de resposta, que são os parâmetros reológicos, utilizou-se as equações linear (equação 2) e quadrática (equação 3) em termos de pseudo-componentes. A significância estatística das equações foi feita através da análise da variância, a nível de 5\% de confiança.

$$
\begin{aligned}
& \hat{Y}=b_{1}^{\prime} X_{1}^{\prime}+b_{2}^{\prime} X_{2}^{\prime}+b_{3}^{\prime} X_{3}^{\prime} \\
& \hat{Y}=b_{1}^{\prime} X_{1}^{\prime}+b_{2}^{\prime} X_{2}^{\prime}+b_{3}^{\prime} X_{3}^{\prime}+b_{1}^{\prime} b_{2}^{\prime} X_{1}^{\prime} X_{2}^{\prime}+b_{1}^{\prime} b_{3}^{\prime} X_{1}^{\prime} X_{3}^{\prime}+b_{2}^{\prime} b_{3}^{\prime} X_{2}^{\prime} X_{3}^{\prime}
\end{aligned}
$$

$\wedge$ $\hat{\mathrm{Y}}=$ estimativa da resposta índice de consistência e índice do comportamento do fluído;

b' = coeficientes da equação (determinados conforme CORNELL [5]);

$\mathrm{X}^{\prime}=$ proporção dos pseudocomponentes.

\section{3 - RESULTADOS E DISCUSSÃO}

\section{1 - Análises físicas e químicas}

A Tabela 2 apresenta os resultados das avaliações físicas e químicas da polpa de manga e dos sucos de laranja e cenoura.

TABELA 2. Caracterização físico-química dos sucos estudados

\begin{tabular}{ccccc}
\hline Parâmetros & Suco Cenoura & Polpa Manga & Suco Laranja \\
\hline pH & 6,44 & 4,28 & 3,88 \\
Acidez & 0,11 & 0,33 & 0,66 \\
Sól. Sol. (Brix) & 4,6 & 17,0 & 12,0 \\
\% Aç. Redutor & 2,5076 & 2,9706 & 4,551 \\
\% Aç. Total & 3,53 & 14,1723 & 9,43 \\
\% Fibras & 0,32 & 0,89 & 0,31 \\
\% Polpa & 1,682 & 29,68 & 3,425 \\
\% Pectato Cálcio & 0,108 & 0,8 & 0,34 \\
\hline
\end{tabular}

Os resultados encontrados para a polpa de manga estão na faixa dos valores encontrados por GUNJAL \& WAGHMARE [7], com exceção do teor de açúcar redutor que foi inferior neste trabalho. As características físico-químicas obtidas para o suco de cenoura foram próximas às determinadas por BAWA \& SAINI [3], exceto no teor de sólidos solúveis e no teor de açúcar total, que foram inferiores aos encontrados pelos autores.

\section{2 - Parâmetros reológicos}

As médias das triplicatas dos parâmetros reológicos índice de consistência (K) e índice de comportamento do fluido (n), para as duas temperaturas, são apresentadas na Tabela 3. O coeficiente de correlação, para todas as formulações foi superior a 0,97 , independente da temperatura.

Verifica-se na Tabela 3 que o índice de comportamento do fluido apresentou valores menores que a unidade, indicando comportamento pseudo-plástico, para 
todas as formulações e temperaturas estudadas. $\mathrm{O}$ maior valor de índice de comportamento e menor valor de índice de consistência foi verificado na formulação 2, a qual apresenta maior proporção de suco de laranja. Apesar de ter sido constatado, de modo geral, um aumento no índice de consistência e redução do índice de comportamento com a elevação da temperatura, isso não implicou no aumento da viscosidade aparente obtida nas taxas de deformação de 50 e $500 \mathrm{~s}^{-1}$, como pode ser verificado através da Tabela 3 . Nesta tabela verifica-se, para as duas taxas de deformação e temperatura de $10^{\circ} \mathrm{C}$, que o maior valor de viscosidade aparente foi atribuída a formulação 1 , que apresenta maior proporção de polpa de manga e os menores valores foram atribuídos às formulações 2 e 3 , justamente onde se tem maior fração de sucos de laranja e cenoura, respectivamente, mostrando que tanto o aumento da fração de laranja ou cenoura pouco contribuíram para o aumento da viscosidade aparente. Esses resultados mostram que a composição química da polpa de manga e sucos teve grande influência sobre a viscosidade aparente. Isso também foi observado por GUNJAL \& WAGHMARE [7] estudando as características de escoamento de polpa, suco e néctar de manga utilizando o modelo de Ostwaldde-Waele. Esses autores verificaram que a viscosidade é influenciada pelo conteúdo de sólidos insolúveis, fibras e pectina pois a viscosidade da polpa foi maior que o suco e esta maior que o néctar. Esses resultados estão de acordo com os encontrados no presente trabalho, pois as maiores viscosidades também foram encontradas nas formulações com maiores proporções de manga, sendo que esta possui os maiores teores de polpa, pectina e sólidos solúveis.

TABELA 3. Valores da viscosidade aparente nas taxas de deformação de 50 e $500 \mathrm{~s}^{-1}$ e dos parâmetros do modelo de Ostwald- De Waele para as diferentes formulações e temperaturas.

\begin{tabular}{|c|c|c|c|c|c|c|c|c|c|c|}
\hline \multirow[t]{2}{*}{ Formulação } & \multicolumn{5}{|c|}{$\mathrm{T}=10^{\circ} \mathrm{C}$} & \multicolumn{5}{|c|}{$\mathrm{T}=60^{\circ} \mathrm{C}$} \\
\hline & $\begin{array}{r}\mathrm{K} \\
\left(\mathrm{Pa}^{*} \mathrm{~s}^{n}\right) \\
\end{array}$ & $n$ & $\mathrm{R}^{2}$ & \multicolumn{2}{|c|}{$\eta_{\mathrm{a}}\left(\mathrm{Pa}^{*} \mathrm{~s}\right)$} & K & $n$ & $\mathrm{R}^{2}$ & \multicolumn{2}{|c|}{$\eta_{\mathrm{a}}\left(\mathrm{Pa}^{*} \mathrm{~s}\right)$} \\
\hline 1 & 2,0195 & 0,4487 & 0,998 & 0,2337 & 0,0656 & 1,9859 & 0,3527 & 0,988 & 0,1579 & 0,0355 \\
\hline 2 & 0,8569 & 0,5078 & 0,996 & 0,1249 & 0,0401 & 1,5696 & 0,3527 & 0,984 & 0,1247 & 0,0281 \\
\hline 3 & 1,0201 & 0,4715 & 0,996 & 0,1290 & 0,0382 & 1,9244 & 0,3106 & 0,990 & 0,1297 & 0,0265 \\
\hline 4 & 1,4879 & 0,4597 & 0,998 & 0,1797 & 0,0518 & 2,2531 & 0,3287 & 0,987 & 0,1630 & 0,0347 \\
\hline 5 & 1,5532 & 0,4502 & 0,998 & 0,1808 & 0,0509 & 2,5674 & 0,3058 & 0,979 & 0,1699 & 0,0343 \\
\hline 6 & 1,1051 & 0,4662 & 0,995 & 0,1369 & 0,0400 & 2,1461 & 0,3006 & 0,979 & 0,1392 & 0,0278 \\
\hline 7 & 1,3892 & 0,4539 & 0,997 & 0,1641 & 0,0466 & 2,6006 & 0,2944 & 0,985 & 0,1646 & 0,0324 \\
\hline 8 & 1,6347 & 0,4567 & 0,997 & 0,1952 & 0,0558 & 2,4159 & 0,3245 & 0,981 & 0,1719 & 0,0363 \\
\hline 9 & 1,0938 & & 0,997 & 0,1419 & 0,0426 & 2,1454 & 0,3176 & 0,986 & 0,1486 & 0,0308 \\
\hline 10 & 1,1215 & 0,4588 & 0,995 & 0,1351 & 0,0388 & 2,2991 & 0,3042 & 0,984 & 0,1511 & 0,0304 \\
\hline
\end{tabular}

$\mathrm{K}=$ Índice de consistência $\left(\mathrm{Pa}^{*} \mathrm{~s}^{\mathrm{n}}\right)$

$\mathrm{n}=$ İndice de comportamento $(-)$
$\eta=$ Viscosidade aparente $\left(\mathrm{Pa}^{*} \mathrm{~s}\right)$

Na temperatura de $60^{\circ} \mathrm{C}$, para as duas taxas de deformação, a viscosidade aparente da formulação 8 foi ligeiramente superior a formulação 1 e, conforme a Tabela 1 , na formulação 8 encontra-se maior fração de suco de laranja e cenoura e menor de polpa de manga que a formulação 1. Esse aumento de viscosidade aparente provavelmente está relacionado com a temperatura dos ensaios que pode ter alterado a estrutura dos constituintes dos sucos ou mesmo concentrado a amostra estu- dada. VIDAL [19] estudando o comportamento reológico de polpa de manga integral, observou que o aumento de temperatura na faixa de 50 a $60^{\circ} \mathrm{C}$, acarretou na diminuição do índice de consistência, aumento do índice de comportamento e diminuição da viscosidade. De acordo com o autor, esse aumento pode ser explicado pela possível gelatinização do amido presente na polpa.

\section{3 - Delineamento experimental}

A superfície de resposta para o parâmetro $\mathrm{K}$, nas temperaturas de 10 e $60^{\circ} \mathrm{C}$, foi melhor representada pelo ajuste linear e quadrático, respectivamente (Figuras 1 $e$ 3). A análise da variância do índice de consistência, para as duas temperaturas, encontra-se na Tabela $4 \mathrm{e}$ pode ser verificado que o ajuste com o modelo linear e quadrático apresentou-se significativo e não foi verificado falta de ajuste devido às replicatas experimentais. $\mathrm{O}$ indice de comportamento do fluido, nas temperaturas de 10 e $60^{\circ} \mathrm{C}$, também foi melhor representado pelos modelos linear e quadrático, respectivamente (Tabela 5). Verifica-se através da Tabela 4 que o aumento da temperatura acarretou, além da utilização de modelo mais elaborado para descrever o índice de consistência, uma redução da significância da equação, apesar de ser significativa ao nível de confiança de 95\%.

TABELA 4. Análise da variância dos modelos de regressão linear e quadrático dos dados de índice de consistência do fluido (K) nas temperaturas de 10 e $60^{\circ} \mathrm{C}$.

\begin{tabular}{c|c|c|c|c|c|c|c|c}
\hline Fonte de & \multicolumn{4}{|c|}{$10^{\circ} \mathrm{C}$} & \multicolumn{4}{c}{$60^{\circ} \mathrm{C}$} \\
\cline { 2 - 10 } Variação & S. Q. & G. L & M. Q. & F & S. Q. & G. L & M. Q. & F \\
\hline Regressão & 3,2514 & 2 & 1,6257 & 107,32 & 2,5971 & 5 & 0,5194 & 15,594 \\
Resíduos & 0,4089 & 27 & 0,0151 & & 0,7994 & 24 & 0,3331 & \\
Falta de Ajuste & 0,0898 & 7 & 0,0128 & 0,804 & 0,0648 & 4 & 0,0162 & 0,4412 \\
Erro Puro & 0,3191 & 20 & 0,0159 & & 0,7346 & 20 & 0,0367 & \\
Total & 3,6603 & 29 & 0,1262 & & 3,3965 & 29 & 0,1171 & \\
\hline
\end{tabular}

TABELA 5. Análise da variância dos modelos de regressão linear e quadrático dos dados de índice de comportamento do fluido (n) nas temperaturas de 10 e $60^{\circ} \mathrm{C}$.

\begin{tabular}{c|cc|c|c|c|c|c|c}
\hline Fonte de & \multicolumn{4}{|c|}{$10^{\circ} \mathrm{C}$} & \multicolumn{4}{c}{$60^{\circ} \mathrm{C}$} \\
\cline { 2 - 10 } Variação & S. Q. & G. L & M. Q. & F & S. Q. & G. L & M. Q. & F \\
\hline Regressão & 0,00062 & 2 & 0,0031 & 12,302 & 0,0109 & 5 & 0,0022 & 12,212 \\
Resíduos & 0,0068 & 27 & 0,0002 & & 0,0043 & 24 & 0,0002 & \\
Falta de Ajuste & 0,0021 & 7 & 0,0003 & 1,2755 & 0,0004 & 4 & 0,0001 & 0,5506 \\
Erro Puro & 0,0047 & 20 & 0,0002 & & 0,0038 & 20 & 0,0002 & \\
Total & 0,013 & 29 & 0,0004 & & 0,0152 & 29 & 0,0005 & \\
\hline
\end{tabular}

Os valores dos coeficientes da equação linear (equação 2) e quadrática (equação 3) para representação de K e n, assim como seus erros padrão, nas duas temperaturas estudadas, em termos de pseudo-componentes e componentes originais, estão nas Tabelas 6, 7 e 8 . Os asteriscos apresentados juntamente com os coeficientes da equação significam que estes são significativos e não são desprezados da equação.

Analisando a equação linear encontrada em função dos pseudo-componentes (Tabela 6), verifica-se que o índice de consistência aumentou com a proporção de 
polpa de manga (X'1) e que o suco de laranja (X'2) exerce o menor efeito no aumento do índice de consistência. Esses resultados mostram que o índice de consistência está diretamente relacionado com a composição das amostras analisadas, pois maiores foram os valores das análises físicas e químicas realizadas com a polpa de manga. Situação contrária foi observada para o índice de comportamento do fluido, que aumenta com a proporção de suco de laranja (X'2) e diminui com o aumento da proporção de polpa de manga (X'1), mostrando uma redução da pseudo-plasticidade da mistura.

TABELA 6. Valores dos coeficientes do modelo linear para o índice de consistência (K) e índice de comportamento do fluido (n), na temperatura de $10^{\circ} \mathrm{C}$.

\begin{tabular}{|c|c|c|c|c|c|c|}
\hline \multirow[b]{2}{*}{ Pseudo-Componentes } & \multicolumn{3}{|c|}{$\mathrm{K}$} & \multicolumn{3}{|c|}{$\mathrm{N}$} \\
\hline & $\mathrm{X}^{\prime} 1$ & $\mathrm{X}^{\prime} 2$ & X'3 & $\mathrm{X}^{\prime} 1$ & $\mathrm{X}^{\prime 2}$ & X'3 \\
\hline Coeficientes da equação & $\underset{*}{2,0202}$ & 0,9143 & 1,0591 & $0,{ }_{*}^{0,419}$ & $\underset{*}{0,4936}$ & $\begin{array}{c}0,4601 \\
*\end{array}$ \\
\hline Erro padrão do coeficiente & 0,0524 & 0,0524 & 0,0524 & 0,0067 & 0,0067 & 0,0067 \\
\hline Componentes Originais & $\mathrm{X} 1$ & $\mathrm{X} 2$ & X3 & $\mathrm{X} 1$ & $\mathrm{X} 2$ & X3 \\
\hline Coeficientes da equação & $\underset{*}{3,0537}$ & $\underset{*}{-2,4757}$ & $-1,7515$ & $\underset{*}{0,4069}$ & $\underset{*}{0,6656}$ & $\underset{*}{0,4979}$ \\
\hline Erro padrão do coeficiente & 0,1205 & 0,3141 & 0,3141 & 0,0155 & 0,0405 & 0,0405 \\
\hline
\end{tabular}

TABELA 7. Valores dos coeficientes do modelo quadrático para o indice de consistência do fluido $(\mathrm{K})$, na temperatura de $60^{\circ} \mathrm{C}$.

\begin{tabular}{|c|c|c|c|c|c|c|}
\hline Pseudo-Componentes & $\overline{X^{\prime} 1}$ & $\mathrm{X}^{\prime 2}$ & $\bar{X} 3$ & $\overline{X^{\prime} 1 X^{\prime} 2}$ & $\overline{X^{\prime} 1 X^{\prime} 3}$ & $\overline{X^{\prime} 2 X^{\prime} 3}$ \\
\hline Coefici & 1,9898 & 1,5651 & 1,9006 & 1,9788 & 2,4882 & 1,70188 \\
\hline Erro & 0,1016 & 0,1016 & 0,1016 & 0,4684 & 0,4684 & 0,4684 \\
\hline Componentes Originais & $\mathrm{X} 1$ & $\mathrm{X} 2$ & $\mathrm{X} 3$ & $\mathrm{X} 1 \mathrm{X} 2$ & $\mathrm{X} 1 \mathrm{X3}$ & $\mathrm{X} 2 \mathrm{X3}$ \\
\hline Coeficientes da equação & $-1,7946$ & $-\underset{*}{-26,688}$ & $-32,65$ & $\underset{*}{49,47}$ & $\underset{\star}{62,204}$ & $\underset{\star}{42,547}$ \\
\hline Erro padrão do coeficiente & 0,8049 & 6,5953 & 6,5953 & 11,709 & 11,709 & 11,709 \\
\hline
\end{tabular}

Para o índice de consistência ajustado com o modelo quadrático (Tabela 7), pode-se observar que a polpa de manga (X'1) e o suco de cenoura (X'3) contribuem para aumentar o parâmetro $\mathrm{K}$ e a presença simultânea dos dois ( $X^{\prime} 1 X^{\prime} 3$ ), acarreta em um aumento significativo do parâmetro reológico. Dentre os sucos estudados, o suco de laranja (X'2) é o que menos contribuiu para o aumento do índice de consistência. O aumento do parâmetro é observado quando reduz-se a fração deste suco e aumenta-se a fração do suco de cenoura e polpa de manga.

TABELA 8. Valores dos coeficientes do modelo quadrático para o índice de comportamento do fluido (n), na temperatura de $60^{\circ} \mathrm{C}$.

\begin{tabular}{|c|c|c|c|c|c|c|}
\hline Pseudo-Componentes & $\mathrm{X}^{\prime} 1$ & $\mathrm{X}^{\prime 2}$ & X'3 & $X^{\prime} 1 X^{\prime} 2$ & $\mathrm{X}^{\prime} 1 \mathrm{X}^{\prime} 3$ & $\overline{X^{\prime} 2 X^{\prime} 3}$ \\
\hline Coeficientes da equação & $\underset{*}{0,3534}$ & $\underset{*}{0,3525}$ & $\underset{*}{0,3126}$ & $-\underset{*}{-0,101}$ & $\begin{array}{c}-0,104 \\
*\end{array}$ & $-0,1275$ \\
\hline Erro $\mathrm{p}$ & 0,0074 & 0,0074 & 0,0074 & 0,0343 & 0,0343 & 0,0343 \\
\hline Componentes Originais & $\mathrm{X} 1$ & $\mathrm{X} 2$ & $\mathrm{X} 3$ & $\mathrm{X} 1 \mathrm{X} 2$ & $\mathrm{X} 1 \mathrm{X3}$ & $\mathrm{X} 2 \mathrm{X} 3$ \\
\hline Coeficientes & $\begin{array}{c}0,5474 \\
*\end{array}$ & $\underset{*}{1,8638}$ & $\begin{array}{c}1,7102 \\
*\end{array}$ & $-2,525$ & $-2,601$ & $-3,188$ \\
\hline Erro padrão do coeficiente & 0,0589 & 0,4829 & 0,4829 & 0,8573 & 0,8573 & 0,8573 \\
\hline
\end{tabular}

Através dos valores dos coeficientes dos pseudocomponentes da equação quadrática, para o índice de comportamento do fluido (Tabela 8), pode ser verificado

que a polpa de manga e o suco de laranja tiveram o mesmo efeito sobre o parâmetro reológico, ou seja, nas formulações onde a fração de manga e laranja foram maiores (formulação 1 e 2), maior foi o valor do índice de comportamento do fluido. Também pode ser verificado através da Tabela 8 que a presença simultânea da polpa de manga e suco de laranja não contribuiu para aumentar o índice de comportamento. Na Tabela 3, temperatura de $60^{\circ} \mathrm{C}$, observa-se que as formulações 1 e 2 obtiveram valores iguais e superiores de índice de comportamento quando comparados às outras formulações.

A representação do ajuste linear para os parâmetros reológicos $\mathrm{K} \mathrm{e} \mathrm{n}$, em termos de pseudo-componentes, é dado através das Figuras 1 e 2 onde observa-se que o índice de consistência aumentou com a fração de polpa de manga e que quanto maior a fração de suco de laranja, menor foi o índice de consistência. Já o índice de comportamento apresentou comportamento contrário ao índice de consistência, pois a medida que aumentou a fração de suco de laranja, houve aumento deste parâmetro.

Índice de Consistência $R^{2}=0,8883$ $\mathrm{K}=2,0202^{*} X^{\prime} 1+0,9143^{*} X^{\prime} 2+1,0591^{*} X^{\prime} 3$

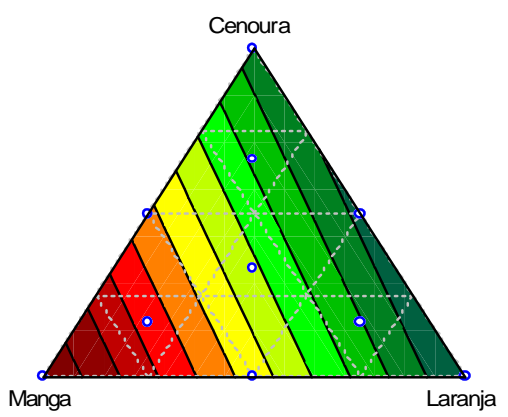

FIGURA 1 - Diagrama ternário da superfície de resposta do modelo linear relativo ao índice de consistência do fluido na temperatura de $10^{\circ} \mathrm{C}$.

Índice de Comportamento $\mathrm{R}^{2}=0,8883$ $n=0,4419^{*} X^{\prime} 1+0,4936^{*} X^{\prime 2} 2+0,4601^{*} X^{\prime} 3$

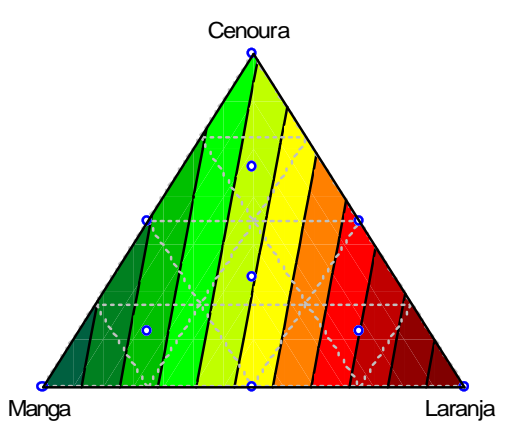

FIGURA 2. Diagrama ternário da superfície de resposta do modelo linear relativo ao índice de comportamento do fluido na temperatura de $10^{\circ} \mathrm{C}$.

A representação do modelo quadrático, em termos de pseudo-componentes, para os dois parâmetros reológicos, encontra-se nas Figuras 3 e 4 . Pode ser 
visualizado na Figura 3 que o aumento na fração de suco de laranja nas formulações deste delineamento acarretou na diminuição do índice de consistência do fluido e o maior valor observado corresponde à formulação 5, onde encontra-se 70\% de polpa de manga, 10\% de suco de laranja e $20 \%$ de suco de cenoura.

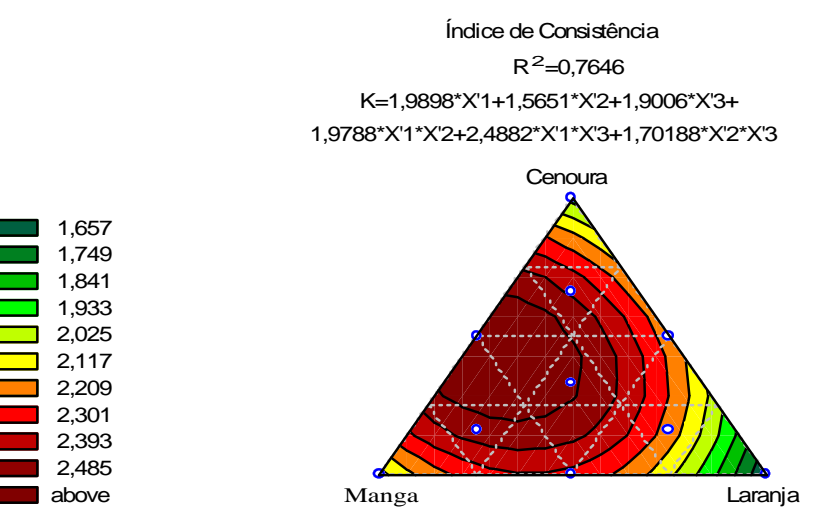

FIGURA 3. Diagrama ternário da superfície de resposta do modelo quadrático relativo ao índice de consistência do fluido na temperatura de $60^{\circ} \mathrm{C}$.
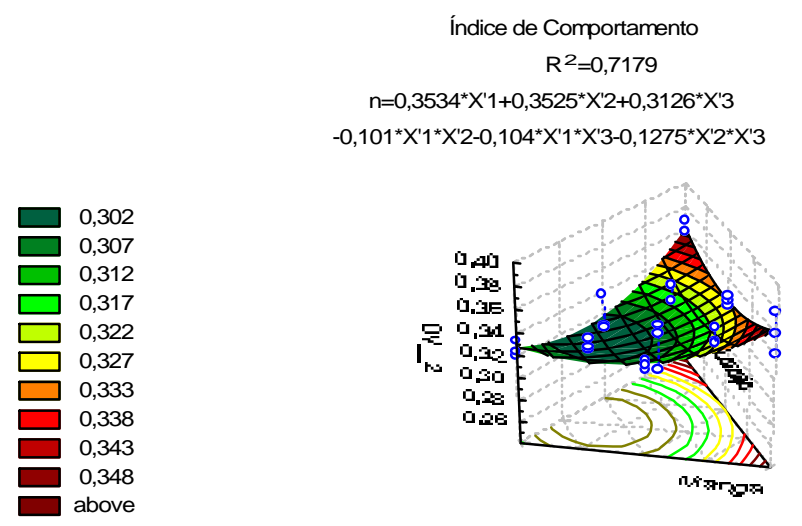

FIGURA 4. Diagrama ternário da superfície de resposta do modelo quadrático relativo ao índice de comportamento do fluido na temperatura de $60^{\circ} \mathrm{C}$.

\section{4 - CONCLUSÕES}

Todas as formulações estudadas, independente da temperatura, apresentaram comportamento pseudoplástico. A viscosidade aparente obtida pelo modelo de Ostwald- De Waele diminuiu com o aumento da taxa de deformação e temperatura, conforme esperado para líquidos. No entanto, se forem observados os parâmetros K do modelo de Ostwald- De Waele, esse parâmetro aumentou com o aumento de temperatura, porém a forma desse aumento é conseqüência da escolha do modelo. O índice de consistência e, principalmente, a viscosidade aparente aumentou com o aumento da polpa de manga mostrando que estes parâmetros são diretamente influenciados pela composição química da polpa. Efeito contrário encontrou-se aumentando a proporção de suco de laranja e cenoura. Quanto maior a proporção desses sucos, menores foram os valores de indice de consistência e viscosidade aparente e maiores os valores do índice de comportamento do fluido.

A elevação da temperatura acarretou na utilização de modelos mais elaborados na predição do comportamento dos parâmetros, pois na temperatura de $10^{\circ} \mathrm{C}$ os dados foram representados por ajuste linear, enquanto na temperatura de $60^{\circ} \mathrm{C}$ o modelo quadrático melhor representou os dados. Os ajustes com os modelos linear e quadrático, para as duas temperaturas estudadas, foram significativos e não foi verificado falta de ajuste devido às replicatas experimentais.

\section{5 - REFERÊNCIAS BIBLIOGRÁFICAS}

[1] AOAC. 1984. Official methods of analysis. Arlington: Association of Official Analytical Chemists, $14 \mathrm{ed}, 1141 \mathrm{p}$.

[2] ASP, N-G.; JOHANSSON, C. G.; HALlMER, H.; SILJSTROM, M. Rapid enzymatic assay of insoluble and soluble dietary fiber. Journal Agric. Food Chemisty, v. 31, p. 476-482, 1983.

[3] BAWA, A. S.; SAINI, P. S. Effect of Method of Preservation on the Storage Quality of Carrot Juice. Indian Food Packer, v. 41, n. 1, p. 42-46, 1987.

[4] BRANCO, I. G. Suco de Laranja Concentrado- Comportamento Reológico a Baixas Temperaturas. Campinas (SP), 1995. 91p. Tese de Mestrado, UNICAMP.

[5] CORNELL, J. A. Experiments with Mixtures: Designs, Models and the Analysis of Mixture Data. 2- ${ }^{\circ}$ ed., Nova York; Wiley, 1990.

[6] CRANDAll, P. G.; CHEN, C. S.; CARTER, R. D. Models for Predicting Viscosity of Orange Juice Concentrate. Food Technology, v. 5, n. 36, p. 245-252, 1982.

[7] GUNJAL, B. B.; WAGHMARE, N. J. Flow Characteristics of Pulp, Juice and Nectar of 'Baneshan' and 'Neelum' Mongoes. Journal of Food Science and Technology, v. 24, p. 20-23, 1987.

[8] LOMBRAÑA, J. I.; DIAS, J. M. Rheological and Chemical Changes in Stored Carrot Juice. Can. Ins. Food Sci. Technol. J., v. 18, n. 3, p. 213-119, 1985.

[9] MANOHAR, B.; RAMAKRISHNA, P.; RAMTEKE, R. S. Effect of Pectin Content on Flow Properties of Mango Pulp Concentrates. Journal of Texture Studies, v. 21, p. 179-190, 1990.

[10] MIZRAHI, S.; BERK, Z. Flow Behaviour of Concentrated Orange Juice: Mathematical Treatment. Journal of Texture Studies, v. 3, n. 1, p. 69-79, 1971.

[11] MIZRAHI, S.; FIRSTENBERG, R. Effect of Orange Composition on Flow Behaviour of Six Fold Concentrate. Journal of Texture Studies, v. 4, n. 6, p. 523-532, 1975.

[12] PELEGRINE, D. H. Comportamento Reológico das Polpas de Manga e Abacaxi. Campinas (SP), 1999. 115p. Tese de Mestrado, UNICAMP.

[13] QUEIROZ, A. J. M. Estudo do comportamento reológico dos sucos de abacaxi e manga. Campinas (SP), 1998. 170p. Tese de Doutorado, UNICAMP.

[14] RANGANNA, S. 1977. Manual of analysis of fruit and vegetable products. Tata McGraw-Hill Publishing Company Limited New Dehi. 634p.

[15] RAO, M. A.; COOley, H. J.; VITAli, A. A. Flow properties of concentrated juices at low temperatures. Food Technology, v. 38, n. 3, p. 113-119, 1984.

[16] RAO, K. L.; EIPESON, W. E.; RAO, P. N. S.; PATWARDHAN, M. V.; RAMANATHAN, P. K.; Rheological Properties of 
Mango Pulp and Concentrates. Journal of Food Science. and Technology, v. 22, n.1, p. 30-33, 1985.

[17] SAEED, A. R. Viscosity of Mango Nectar as Related to Pectic Substances. Journal of Food Science, v. 40, p. 203-204, 1975.

[18] VIDAL, J. R. M. B.. Estudo Reológico do Suco de Manga - Efeito dos Sólidos Solúveis e Insolúveis. Campinas (SP), 1996. 79p. Tese de Mestrado, UNICAMP.
[19] VIDAL, J. R. M. B. Comportamento reológico da polpa de manga. (SP), 2000. 159 p. Tese de Doutorado, UNICAMP.

[20] VITALI, A. A. Comportamento Reológico do Suco de Laranja Concentrado Congelado à Baixas Temperaturas. 1983. Tese de Doutorado, Faculdade de Engenharia Química, EPUSP. 